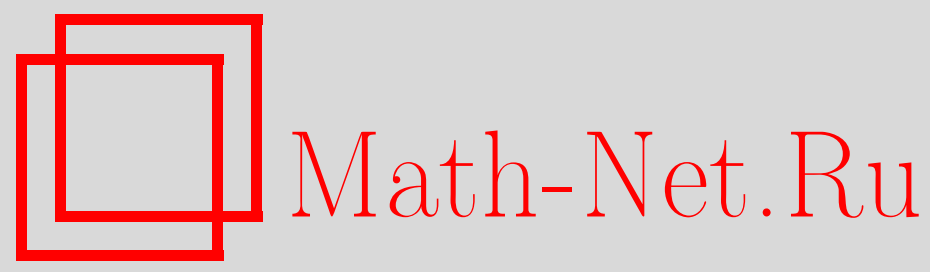

С. А. Богатый, Д. Л. Гонсалвес, Е. А. Кудрявцева, Х. Цишанг, Минимальное число прообразов при отображениях поверхностей, Матем. заметки, 2004, том 75, выпуск 1, 1319

DOI: https://doi.org/10.4213/mzm2

Использование Общероссийского математического портала Math-Net.Ru подразумевает, что вы прочитали и согласны с пользовательским соглашением http://www . mathnet.ru/rus/agreement

Параметры загрузки:

IP : 3.89.185.249

26 апреля 2023 г., 14:36:17 


\title{
МИНИМАЛЬНОЕ ЧИСЛО ПРООБРАЗОВ ПРИ ОТОБРАЖЕНИЯХ ПОВЕРХНОСТЕЙ
}

\author{
С. А. Богатый, Д. Л. Гонсалвес, Е. А. Кудрявцева, Х. Цишанг
}

\begin{abstract}
Вычислено минимаљное число корней уравнения $f(x)=c$ для отображений $f$ из заданного гомотопического класса отображений между замкнутыми (не обязательно ориентируемьми) поверхностями.

Библиографой: 20 названий.
\end{abstract}

1. Введение. Пусть $M_{1}$ и $M_{2}$ - замкнутые многообразия, $f: M_{1} \rightarrow M_{2}$ - непрерывное отображение и $c \in M_{2}$ - точка в образе. Минимальным числом корней для отображения $f$ назьвается

$$
M R[f]=\min _{\tilde{f} \simeq f}\left|\tilde{f}^{-1}(c)\right|,
$$

где “” означает гомотопность. Под задачей корней понимается задача вычисления $M R[f]$. Более общей является задача совпадения - определить минимальное число точек совпадения

$$
M C\left[f_{1}, f_{2}\right]=\min _{\tilde{f}_{1} \simeq f_{1}, \tilde{f}_{2} \simeq f_{2}}\left|\operatorname{coin}\left(\tilde{f}_{1}, \tilde{f}_{2}\right)\right|,
$$

где $\operatorname{coin}\left(\tilde{f}_{1}, \tilde{f}_{2}\right)=\left\{x \in M_{1} \mid \tilde{f}_{1}(x)=\tilde{f}_{2}(x)\right\}$, для двух непрерывных отображений $f_{1}, f_{2}: M_{1} \rightarrow M_{2}$. Другим широко известным специальньм случаем задачи совпадения является задача неподвижных точек (где $M_{1}=M_{2}$ и $f_{2}=\mathrm{id}_{M_{1}}$ ), которой посвящено много исследований.

В случае, когда $M_{1}$ и $M_{2}$ - многообразия одной размерности, известно много глубоких результатов. В частности, для всякой пары отображений $f_{1}, f_{2}: M_{1} \rightarrow M_{2}$ минимальное число совпадения $M C\left[f_{1}, f_{2}\right]$ не меньше числа совпадения Нильсена $N C\left[f_{1}, f_{2}\right]$, т.е. числа существенных классов совпадения; под "существенным" понимается класс совпадения с ненулевым индексом:

$$
M C\left[f_{1}, f_{2}\right] \geqslant N C\left[f_{1}, f_{2}\right] .
$$

Если размерность $n$ многообразий отлична от 2 , то проблему совпадения решили для пары произвольных отображений в ориентируемом случае Х. Ширмер [1] и в неориентируемом случае Р. Добренко и Ю. Езерски [2], доказав справедливость свойства Векена

Работа выполнена в ноябре-декабре 2000 г. во время визита первого автора на Fakultät für Mathematik, Ruhr-Universität Bochum. Этому автору приятно выразить благодарность Ruhr-Universität Bochum за гостеприимство. Визит был поддержан DFG-проектом "Niedrigdimensionale Topologie und geometrisch-topologische Methoden in der Gruppentheorie". Второй автор пользовался поддержкой FAPESP по проекту "Projeto Temático Geometria e Topologia".

(C) С.А. БоГАтый, Д.Л. ГОНСАлвеС, Е.А. КудрявцевА, Х. ЦишАнГ 2004 
для пары произвольных отображений, т.е. доказав, что минимальное число совпадения равно числу совпадения Нильсена. Если $n=2$ и $M_{2}$ является 2-мерной сферой $S^{2}$ или проективной плоскостью $\mathbb{P}^{2}$, то справедливость свойства Векена доказана в [3, предложение 2.2] и [4, теорема 4.10].

В отличие от случая $\operatorname{dim} M_{j} \geqslant 3$ двумерные многообразия не имеют достаточно свободы для осуществления "освобождающих" деформаций, но в отличие от случая $\operatorname{dim} M_{j}=1$ они недостаточно просты и существуют отображения, для которых минимальное число корней $M R[f]$ и число Нильсена корней $N R[f]=N C[f, c]$ не совпадают. Первьй пример такого отображения замкнутой ориентируемой поверхности рода 2 в тор построил Х. Хопф [5], см. [6, пример 5.10].

Пусть $A(f)$ обозначает абсолютную степень отображения $f: M_{1} \rightarrow M_{2}$, см. [7] или $[5]$, и пусть $\ell(f)=\left[\pi_{1}\left(M_{2}\right): f_{\#}\left(\pi_{1}\left(M_{1}\right)\right)\right]$. Рассмотрим проблему корней для $f$. Согласно Р. Бруксу [8, (3.21)] все классы Нильсена корней имеют одинаковый индекс $A(f) / \ell(f)$ и число этих классов равно $N R[f]=\ell(f)<\infty$, если $A(f)>0$, см. также [9, предложение 2.2 и лемма 5.7].

В размерности $2 \mathrm{X}$. Кнезер [10] показал, что $M R[f]=0$, если $A(f)=0$, и $M R[f] \leqslant$ $\ell(f)=A(f)$, если отображение $f$ не является ориентирующе-правильным и $A(f)>0$. Согласно [9, теорема 4.6]

$$
M R[f]=\ell(f) \Longleftrightarrow \ell(f) \geqslant \chi\left(M_{1}\right)+\left(1-\chi\left(M_{2}\right)\right) A(f),
$$

если $A(f)>0$ (доказательство опирается на сильньй результат Д. Габаи и В. Х. Казеса [11]); согласно $\left[9\right.$, теорема 2.5] $M R[f] \geqslant \chi\left(M_{1}\right)+\left(1-\chi\left(M_{2}\right)\right) A(f)$, если отображение $f$ является ориентирующе-правильным и $A(f)>0$.

Данная статья является продолжением [3], [9], [12] и мы доказываем следующий результат.

Теорема 1.1. Пусть $f: M_{1} \rightarrow M_{2}$ - непрерывное отображение замкнутых поверхностей абсолютной степени $A(f)$. Тогда

(a) если $A(f)=0$, то $M R[f]=N R[f]=0$;

(b) если $A(f)>0$, то для $\ell(f)=\left[\pi_{1}\left(M_{2}\right): f_{\#}\left(\pi_{1}\left(M_{1}\right)\right)\right]$

$$
M R[f]=\max \left\{\ell(f), \chi\left(M_{1}\right)+\left(1-\chi\left(M_{2}\right)\right) A(f)\right\}, \quad N R[f]=\ell(f) .
$$

2. Первые шаги доказательства. Через $S_{g}$ мы обозначаем замкнутую ориентируемую поверхность рода $g$; ее эйлерова характеристика равна $2-2 g$. Через $N_{g}$ мы обозначаем замкнутую неориентируемую поверхность, полученную из 2-сферы приклеиванием $g+1$ листа Мёбиуса; ее эйлерова характеристика равна $1-g$, и существует ориентирующее (двулистное) накрытие $S_{g} \rightarrow N_{g}$.

Согласно упомянутьм во введении результатам нам осталось только рассмотреть ситуацию, когда $M_{2} \not \approx S_{0}, N_{0}, A(f)>0$ и отображение $f$ является ориентирующе-правильным. При этих условиях индекс $\ell(f)$ подгруппы $f_{\#}\left(\pi_{1}\left(M_{1}\right)\right)$ в $\pi_{1}\left(M_{2}\right)$ конечен.

ПРЕДЛОЖЕНИЕ 2.1. Пусть $f: M_{1} \rightarrow M_{2}$ - непрерывное отображсение замкнутых поверхностей, $M_{2} \not S_{0}, N_{0}$, и абсолютная степень $A(f)>0$. Тогда для $\ell(f)=\left[\pi_{1}\left(M_{2}\right): f_{\#}\left(\pi_{1}\left(M_{1}\right)\right)\right]$

$$
M R[f]=\max \left\{\ell(f), \chi\left(M_{1}\right)+\left(1-\chi\left(M_{2}\right)\right) A(f)\right\} .
$$


ДокАЗАТЕльство. Так как отображение $f$ может быть поднято на $\ell(f)$-листное (неразветвленное) накрытие $M_{2}$, отвечающее подгрупше $f_{\#}\left(\pi_{1}\left(M_{1}\right)\right) \subset \pi_{1}\left(M_{2}\right)$, то справедливо неравенство $M R[f] \geqslant \ell(f)$.

Нам необходимо рассмотреть различные случаи и мы закончим доказательство предложения 2.1 (и тем самьп теоремы 1.1) только в разделе 4. Напомним, что отображение $f: M_{1} \rightarrow M_{2}$ назьвается ориентирующе-правильным, если замкнутые кривые, сохраняющие ориентацию, переходят при $f$ в кривые, сохраняющие ориентацию, а кривые, обращающие ориентацию, переходят в кривые, обращающие ориентацию, см. также [13].

СлучАй 2.2. Пусть $M_{1}=S_{h}, M_{2}=S_{g}$. В этой ситуации утверждение предложения 2.1 совпадает с [2, теорема 3.3$]$.

СлучАй 2.3. Во всех оставшихся случаях по крайней мере одна поверхность неориентируема. Как уже упоминалось раньше, нам необходимо рассматривать только ориентирующе-правильные отображения. В частности, случай $M_{1}=N_{h}$ и $M_{2}=S_{g}$ полностью сделан. Далее, как уже упоминалось вьше, если $\ell(f) \geqslant \chi\left(M_{1}\right)+\left(1-\chi\left(M_{2}\right)\right) A(f)$, то утверждение следует из [9, теорема 4.3]. Итак, в двух оставшихся случаях нам надо только рассмотреть ориентирующе-правильные отображения $f$ c

$$
\ell(f)<\chi\left(M_{1}\right)+\left(1-\chi\left(M_{2}\right)\right) A(f) .
$$

СлучАй 2.4. Пусть $M_{1}=S_{h}, M_{2}=N_{g}$. Отображение $f$ может быть поднято на двулистное ориентирующее накрытие $p: S_{g} \rightarrow N_{g}$ до отображения $\bar{f}: M_{1}=S_{h} \rightarrow S_{g}$ c $f=p \circ \bar{f}$. Если $d=|\operatorname{deg} \bar{f}|$, то мы имеем $A(f)=2 d$ и $\ell(f)=2 \ell(\bar{f})$. Пусть $p^{-1}(c)=$ $\left\{c_{1}, c_{2}\right\}$. Понятно, что число

$$
A(f) \chi\left(M_{2}\right)-\chi\left(M_{1}\right)=2 d \chi\left(N_{g}\right)-\chi\left(S_{h}\right)=d \chi\left(S_{g}\right)-\chi\left(S_{h}\right)
$$

четно и согласно неравенству Кнезера неотрицательно (см. [9, предложение 1.6]). В соответствии с неравенством (1) мы найдем два таких числа $\mu_{1}, \mu_{2}$, что

$$
\ell(f)=2 \ell(\bar{f})<2 d-\left(d \chi\left(S_{g}\right)-\chi\left(S_{h}\right)\right)=\mu_{1}+\mu_{2} \quad \text { c } \ell(\bar{f}) \leqslant \mu_{i} \leqslant d .
$$

Согласно [3, теорема 5.3] существует такое $d$-листное разветвленное накрытие $\tilde{f}: S_{h} \rightarrow$ $S_{g}$, что

$$
\left|\tilde{f}^{-1}\left(c_{1}\right)\right|=\mu_{1},\left|\tilde{f}^{-1}\left(c_{2}\right)\right|=\mu_{2} \quad \text { c } \quad \tilde{f}_{\#}\left(\pi_{1}\left(S_{h}\right)\right)=\bar{f}_{\#}\left(\pi_{1}\left(S_{h}\right)\right) .
$$

Согласно теореме Габаи-Казеса [11] существует такой гомеоморфизм $q: S_{h} \rightarrow S_{h}$, что $\bar{f} \simeq \tilde{f} \circ q$. Отображение $p \circ \tilde{f} \circ q$ является искомьм.

СлучАЙ 2.5. Пусть $M_{2}=N_{g}, M_{1}=N_{h}$. Этот случай будет рассмотрен в разделе 4 .

\section{3. Построение разветвленных накрытий над неориентируемыми поверх-} ностями. Перед окончанием доказательства сформулируем и докажем некоторые вспомогательные результаты, представляющие и самостоятельньй интерес. В последуюшем мы пишем операцию перестановки множества $\{1,2, \ldots, d\}$ с правой стороны от множества. Пусть $\Sigma_{d}$ обозначает симметрическую группу на $d$ символах. Прямым вычислением получается 
Лемма 3.1. Пусть $d \geqslant 3 k+1, k \geqslant 0 u \alpha=(d, d-1, \ldots, 3,2,1)$,

$$
\beta_{k}=(1,2,4,5,7, \ldots, 3 k-2,3 k-1,3 k+1,3 k+2,3 k+3, \ldots, d)(3) \ldots(3 k) .
$$

Перестановка $\gamma=\beta_{k}^{2} \alpha^{2}$ имеет $d-2 k$ независимых ииклов; более точно,

$$
\gamma=(1,2,3)(4,5,6) \ldots(3 k-2,3 k-1,3 k)(3 k+1) \ldots(d) .
$$

Лемма 3.2. Подгруппа $\left\langle\alpha, \beta_{k}\right\rangle$ группь $\Sigma_{d}, \quad k \geqslant 1$, примитивна, т.е. не существует собственного разбиения множества $\{1,2, \ldots, d\}$, инвариантного относительно действия $\left\langle\alpha, \beta_{k}\right\rangle$.

ДокаЗАтельСтво. Пусть $P_{1}, \ldots, P_{q}$ - разбиение множества $\{1,2, \ldots, d\}$, инвариантное относительно действия $\left\langle\alpha, \beta_{k}\right\rangle$. Согласно [3, лемма 5.14] инвариантность относительно $\alpha^{-1}$ влечет, что

$$
d=q \cdot \bar{d} \quad \text { и } \quad P_{i}=\{i+j q \mid 0 \leqslant j \leqslant \bar{d}-1\}
$$

после некоторой перенумерации множеств. Покажем, что при $1<q<d$ такое разбиение не является инвариантным при действии перестановки $\gamma$.

Случай $q \geqslant 3 k-1$. Здесь $2 \in P_{2}$ и $2+q \in P_{2}$, но $(2) \gamma=3 \notin P_{2}$ и $(2+q) \gamma=2+q \in P_{2}$; следовательно, разбиение не инвариантно при действии $\left\langle\alpha, \beta_{k}\right\rangle$.

Случай $q \leqslant 3 k$. Здесь $q \in P_{q}$ и $d \in P_{q}$, но $(q) \gamma$ это $q+1$ или $q-2$. Таким образом, $(q) \gamma \notin P_{q}$ в противоречии с $(d) \gamma \in P_{q}$. Для $q=2,(2) \gamma=3 \in P_{1}$.

Tеорема 3.3. Для всякого $d \geqslant 2$ и всякого множества $m$ точек $c_{1}, \ldots, c_{m} \in N_{g}$, где $g>0$, с такими ассоциированными цельции числами $\mu_{1}, \ldots, \mu_{m}$, что

$$
1 \leqslant \mu_{i} \leqslant d-1 \forall i \in\{1, \ldots, m\} \quad u \quad m d-\sum_{i=1}^{m} \mu_{i} \equiv 0 \quad \bmod 2,
$$

существует такое примитивное с множеством ветвления $B_{\varphi}=\left\{c_{1}, \ldots, c_{m}\right\}$, что $\left|\varphi^{-1}\left(c_{i}\right)\right|=\mu_{i}$ для $1 \leqslant i \leqslant m$.

ДоказАТЕЛьство. Наше доказательство основано на теореме Гурвица существования разветвленных накрытий [14], неориентируемьй вариант которой можно найти в [15, теорема 2.1] или [16, лемма 2.1].

Рассмотрим сначала случай $d=p_{1} \cdot \ldots \cdot p_{t}$ с простыми $2 \leqslant p_{1} \leqslant p_{2} \leqslant \cdots \leqslant p_{t}$ и $\mu_{i} \leqslant\left(p_{1}-1\right) p_{2} \cdot \ldots \cdot p_{t}$ для некоторого $i \in\{1, \ldots, m\}$. Согласно теореме Гурвица существует такое разветвленное накрытие $\varphi: M \rightarrow N_{g}$ порядка $d$, что множество $B_{\varphi}$ точек ветвления $\varphi$ состоит из $m$ точек $c_{1}, \ldots, c_{m}$, с разбиениями $A_{1}, \ldots, A_{m}$, где $A_{i}=$ $\left(d-\mu_{i}+1,1,1, \ldots, 1\right)$ и $A_{j}$ являются произвольными разбиениями $d$ на $\mu_{j}$ слагаемых для $j \neq i, 1 \leqslant j \leqslant m$. Согласно [3, следствие 5.11] разветвленное накрытие $\varphi$ примитивно.

Осталось рассмотреть случаи с $2 \mu_{i}>d$. Тогда для $k=[(d-1) / 3]$ мы имеем такое число $k \geqslant 1$, что $d \geqslant 3 k+1$ и $d>\mu_{i} \geqslant d-2 k$. В [3] в доказательстве предложения 5.16 (случаи 2 и 3 ) точкам $c_{1}, \ldots, c_{m}$ приписаны такие перестановки $\gamma_{1}, \ldots, \gamma_{m} \in \Sigma_{d}$ с $\mu_{1}, \ldots, \mu_{m}$ циклами, соответственно, что

$$
\gamma_{1} \cdot \ldots \cdot \gamma_{m}=(123)(456) \ldots(3 r-2,3 r-1,3 r)(3 r+1) \ldots(d-1)(d)
$$


для некоторого $r \in\{1, \ldots, k\}$. Представление $\pi_{1}\left(N_{g} \backslash\left\{c_{1}, \ldots, c_{m}\right\}, *\right)$ в $\Sigma_{d}$ зададим сопоставлением перестановок $\gamma_{1}, \ldots, \gamma_{m}$ каноническим образующим, соответствующим точкам $c_{1}, \ldots, c_{m}$, и $\alpha_{0}=\beta_{r}, \alpha_{1}=\alpha, \alpha_{2}=\cdots=\alpha_{g}=$ id для оставшихся $g+1$ образующих. Согласно лемме 3.2 и [3, предложение 5.6] $d$-листное разветвленное накрытие $\varphi: \widetilde{M} \rightarrow N_{g}$ (определяемое согласно теореме Гурвища по построенному представлению) примитивно.

Так как в обоих случаях разветвленное накрытие примитивно и (как всякое разветвленное накрытие) является ориентирующе-правильньм, то накрьвающеепространство $\widetilde{M}$ является неориентируемой замкнутой поверхностью $N_{\tilde{g}}$.

С использованием неразветвленного накрытия, отвечающего данной подгруппе $\pi_{1}\left(N_{g}\right)$, теорема 3.3 может быть обобщена на непримитивную ситуацию; для детального доказательства см. [3, следствие 5.18].

СлЕДСТВИЕ 3.4. Пусть $N_{g}$ - неориентируемая замкнутая поверхность, отличная от проективной плоскости, т.е. $g \geqslant 1$, и пусть $H \subset \pi_{1}\left(N_{g}, *\right)$ - подгруппа конечного индекса $\ell$. Для всякого иелого числа $d \geqslant \ell+1 c d \equiv 0 \bmod \ell$ и всякого множсества $m$ точек $c_{1}, \ldots, c_{m} \in N_{g}$ с такими ассочиированными числами $\mu_{1}, \ldots, \mu_{m}$, чmo

$$
\ell \leqslant \mu_{i} \leqslant d-1 \quad \text { для } \quad 1 \leqslant i \leqslant m \quad u \quad \mu_{1}+\cdots+\mu_{m} \equiv m d \quad \bmod 2,
$$

существует такое d-листное разветвленное накрытие $\varphi: \widetilde{M} \rightarrow N_{g}$ замкнутой поверхности $\widetilde{M}$ на $N_{g}$ с мнохсеством ветвления $B_{\varphi}=\left\{c_{1}, \ldots, c_{m}\right\}$, что $\varphi_{\#}\left(\pi_{1}(\widetilde{M}, \tilde{*})\right)=H$ для некоторой точки $\tilde{*} \in \varphi^{-1}(*) u\left|\varphi^{-1}\left(c_{i}\right)\right|=\mu_{i}$ для $1 \leqslant i \leqslant m$.

Поверхность $\widetilde{M}$ ориентируема тогда и только тогда, когда $H$ содержится $в$ подгруппе индекса 2, содержащей гомотопические классы всех сохраняющих ориентацию путей, т.е. $H \subset p_{\#}\left(\pi_{1}\left(S_{g}\right)\right)$, где $p: S_{g} \rightarrow N_{g}$ - двулистное ориентирующее накрытие $N_{g}$.

4. Окончание доказательства теоремы 1.1. Остался случай $M_{1}=N_{h}, M_{2}=$ $N_{g}$ с $g \geqslant 1$. Из [9, теорема $\left.2.5(\mathrm{c})\right]$ вытекает, что число $A(f) \chi\left(N_{g}\right)-\chi\left(N_{h}\right)$ четно. Кроме того, следуюшие рассуждения дают неравенства

$$
0<A(f) \chi\left(N_{g}\right)-\chi\left(N_{h}\right)<A(f)-\ell(f) .
$$

Правое неравенство вытекает из (1). Число $A(f) \chi\left(N_{g}\right)-\chi\left(N_{h}\right)$ неотрицательно согласно неравенству Кнезера (как в случае 2.5 ). Оно не зануляется, ибо иначе $f$ будет гомотопно $A(f)$-листному накрытию, что влечет $A(f)=\ell(f)$ в противоречии с (1). Согласно следствию 3.4 существует такое $A(f)$-листное разветвленное накрытие $\varphi: \widetilde{M} \rightarrow N_{g}$, что

$$
B_{\varphi}=\{c\}, \quad \ell(f)<\left|\varphi^{-1}(c)\right|=A(f)-\left(A(f) \chi\left(N_{g}\right)-\chi\left(N_{h}\right)\right)<A(f)
$$

и

$$
\varphi_{\#}\left(\pi_{1}(\widetilde{M}, \tilde{*})\right)=f_{\#}\left(\pi_{1}\left(N_{h}, *_{1}\right)\right)=H .
$$

Так как $f$ является ориентирующе-правильным отображением, а $M_{1}$ неориентируемо, то $H$ не может содержаться в подгрупе $p_{\#}\left(\pi_{1}\left(S_{g}\right)\right)$ классов сохраняющих ориентацию кривьх и, следовательно, $\widetilde{M}$ неориентируема. Для эйлеровых характеристик мы имеем

$$
\chi(\widetilde{M})=A(f) \cdot \chi\left(N_{g}\right)-\left(A(f)-\left|\varphi^{-1}(c)\right|\right)=\chi\left(N_{h}\right)
$$


и, таким образом, $\widetilde{M}$ - неориентируемая поверхность, гомеоморфная $N_{h}$. Согласно теореме Габаи-Казеса [11] существует такой гомеоморфизм $q: N_{h} \rightarrow \widetilde{M}$, что $f$ и $\varphi \circ q$ гомотопны. Теперь утверждение следует из

$$
\begin{aligned}
M R[f] \leqslant\left|\left(q^{-1} \circ \varphi^{-1}\right)(c)\right| & =A(f)-\left(A(f) \chi\left(N_{g}\right)-\chi\left(N_{h}\right)\right) \\
& =\chi\left(N_{h}\right)+\left(1-\chi\left(N_{g}\right)\right) A(f) .
\end{aligned}
$$

ЗАмЕчАния. (а) Для $g \geqslant 3$ в теореме 3.3 и следствии 3.4 всякая система $m$ разбиений числа $d$ на $\mu_{1}, \ldots, \mu_{m}$ слагаемых, соответственно, может быть реализована разветвленным накрытием с данным образом фундаментальной группы.

(b) Опираясь на $[4$, леммы $1.2,3.1]$ или [3, фундаментальная лемма 8.3] или [9, леммы 5.5, 5.7, 6.3], наши результаты можно применить к решению некоторых квадратических уравнений в свободных группах.

(c) Так как тор $S_{1}$ является топологической группой, то минимальное число совпадения $M C\left[f_{1}, f_{2}\right]$ для всякой пары отображений $f_{1}, f_{2}: M_{1} \rightarrow M_{2}=S_{1}$ совпадает с минимальным числом корней $M R\left[f_{1} \overline{f_{2}}\right]$, где $\overline{f_{2}}(x)=\left(f_{2}(x)\right)^{-1}, x \in M_{1}$, является обратным элементом к $f_{2}(x)$ в $S_{1}$, см. [16].

(d) Техника работы Добренко-Езерского [2] сводит вычисление минимального числа совпадения $M C\left[f_{1}, f_{2}\right]$ двух произвольных отображений $f_{1}, f_{2}: M_{1} \rightarrow N_{1}$ в бутылку Клейна к соответствующему вычислению для двух отображений в тор $S_{1}$.

(е) Техника, развитая в работах [3], [9], [12] и этой статье, позволяет вычислить минимальньй прообраз множества $\left\{c_{1}, \ldots, c_{m}\right\}$ из $m$ точек $M_{2}$, т.е. вычислить минимальное число пересечения МакКорда [18] в случае отображения 2-поверхности и вложения 0 -многообразия, состоящего из $m$ точек, в некоторое (возможно другое) 2-многообразие:

$$
\begin{aligned}
M R_{m}[f] & :=\min _{\tilde{f} \simeq f}\left|\tilde{f}^{-1}\left\{c_{1}, \ldots, c_{m}\right\}\right| \\
& =\max \left\{m \ell(f), \chi\left(M_{1}\right)+\left(m-\chi\left(M_{2}\right)\right) \cdot A(f)\right\} .
\end{aligned}
$$

(f) В работах [19], [20] авторы полностью решили задачу существования примитивного разветвленного накрытия с заданными порядками ветвлений. То есть получен более сильный вариант теоремы 3.3, в котором реализуются не только заданные количества прообразов точек ветвления, но и заданные порядки ветвления в этих прообразах.

\section{СПИСОК ЦИТИРОВАННОЙ ЛИТЕРАТУРЫ}

[1] Schirmer H. Mindestzahlen von Koinzidenzpunkten // J. Reine Angew. Math. 1955. V. 194. P. 659-671.

[2] Dobreńko R., Jezierski J. The coincidence Nielsen number on non-orientable manifolds // Rocky Mountain J. Math. 1993. V. 23. P. 67-87.

[3] Bogatyi S., Gonçalves D. L., Zieschang H. The minimal number of roots of surface mappings and quadratic equations in free groups // Math. Z. 2001. V. 236. P. 419-452.

[4] Jezierski J. The least number of coincidence points on surfaces // J. Australian Math. Soc. Ser. A. 1995. V. 58. P. 27-38.

[5] Hopf H. Zur Topologie der Abbildungen von Mannigfaltigkeiten. II // Math. Ann. 1930. V. 102. P. 562-623.

[6] Богатый С. А., Гонсалвес Д. Л., Цишанг Х. Теория совпадения: Проблема минимизации // Тр. МИАН. 1999. Т. 225. С. 52-86.

[7] Epstein D. B. A. The degree of a map // Proc. London Math. Soc. (3). 1966. V. 16. P. 369-383. 
[8] Brooks R. B. S. Certain subgroups of the fundamental group and the number of roots of $f(x)=a / /$ J. Amer. Math. Soc. 1973. V. 95. P. 720-728.

[9] Gonçalves D. L., Kudryavtseva E., Zieschang H. Roots of mappings on nonorientable surfaces and equations in free groups // Manuscripta Math.. V. 107. P. 311-341.

[10] Kneser H. Die kleinste Bedeckungszahl innerhalb einer Klasse von Flächenabbildungen // Math. Ann. 1930. V. 103. P. 347-358.

[11] Gabai D., Kazez W. H. The classification of maps of nonorientable surfaces // Math. Ann. 1988. V. 281. P. $687-702$.

[12] Gonçalves D. L., Zieschang H. Equations in free groups and coincidence of mappings on surfaces // Math. Z. 2001.

[13] Olum P. Mappings of manifolds and the notion of degree // Ann. of Math. 1953. V. 58. P. $458-480$.

[14] Hurwitz A. Über Riemannische Fläche mit gegebenen Verzweigungs-punkten // Math. Ann. 1891. V. 39. P. 1-60.

[15] Ezell C. L. Branched point structure of covering maps onto nonorientable surfaces // Trans. Amer. Math. Soc. 1978. V. 243. P. 123-133.

[16] Brooks R., Wong P. On changing fixed points and coincidences to roots // Proc. Amer. Math. Soc. 1992. V. 115. P. 527-533.

[16] Edmonds A. L., Kulkarni R. S., Stong R. E. Realizability of branched coverings of surfaces // Trans. Amer. Math. Soc. 1984. V. 282. P. 773-790.

[18] McCord C. K. A Nielsen theory for intersection numbers // Fund. Math. 1997. V. 152. P. 117-150.

[19] Bogatyi S., Gonçalves D. L., Kudryavtseva E., Zieschang H. Realization of primitive branched coverings over closed surfaces // Submitted to Kluwer Academic Publishers.

[20] Bogatyi S., Gonçalves D. L., Kudryavtseva E., Zieschang H. Realization of primitive branched coverings over closed surfaces following the Hurwitz approach // Central Europ. J. of Math. 2003. V. 2. P. 184-197.

(Богатый С. А.) Московский государственный

Поступило

университет им. М. В. Ломоносова

15.02 .2001

E-mail: bogatyi@mech.math.msu.su

(Gonçalves Daciberg L.) Departamento de Matemática - IME-USP

Исправленный вариант

Agência Cidade de São Paulo

E-mail: dlgoncal@ime.usp.br

(Кудрявцева Е.А.) Московский государственный

университет им. М. В. Ломоносова и

Ruhr-Universität Bochum

E-mail: eakudr@mech.math.msu.su;

elena.kudrjawzewa@ruhr-uni-bochum.de

(Zieschang H.) Ruhr-Universität Bochum

E-mail: Heiner.Zieschang@ruhr-uni-bochum.de 